\title{
Some Boundary Value Problems Involving Plasma Media
}

\author{
James R. Wait ${ }^{1}$
}

(November 3, 1960)

\begin{abstract}
A plasma, consisting of a neutral mixture of electrons, ions and molecules, in the presence of a constant magnetic field $H_{0}$, possesses a dielectric constant which is in the form of a tensor. Exact solutions of boundary value problems involving such media are obtained for twodimensional configurations. Explicit results are given for the reflection coefficients of stratified plasma in planar and cylindrical geometry.
\end{abstract}

\section{Introduction}

A neutral mixture of positive (and negative) ions and electrons is often described as a plasma. For example, flames, gaseous discharges, strong shock waves, and the ionosphere are various kinds of plasma. The behavior of electromagnetic waves within and in the vicinity of plasma is of great current interest.

Mainly because of the presence of the free electrons the plasma is a dielectric. The collisions of electrons with the ions cause dissipation of energy and thus the dielectric is lossy. A radio wave propagating through the plasma is thus expected to be attenuated. Furthermore, in the presence of a steady magnetic field, the plasma becomes anisotropic so that the dielectric constant is of tensor form and thus, in general, propagation is not reciprocal.

It is the purpose of the present paper to consider the interaction of electromagnetic waves and plasma for a special class of two-dimensional problems. The geometry is chosen so the wave propagation is essentially transverse to the longitudinal axis. Such a restriction permits exact solutions of a number of relevant boundary value problems to be obtained. Since radio waves, particularly microwaves, are being currently employed as a diagnostic tool in probing plasma, it appears that results given below can find application.

An excellent introduction to the theory of propagation of electromagnetic waves in plasma has been given recently by Whitmer [1]. ${ }^{2}$ A more comprehensive treatment is found in a recent monograph by Ratcliffe [2]. The dielectric behavior and the molecular properties of a plasma have been dealt with by a number of authors [3-6]. In particular, Spitzer [7], has given a very thorough discussion for fully ionized gases.

\section{Dielectric Properties of a Plasma}

Since the problems to be discussed in the following sections deal with two-dimensional geometry, it is desirable to take the $z$-axis of the Cartesian or the cylindrical coordinate system in the direction of the applied magnetic field $\vec{H}_{0}$. In this case the dielectric displacement $\vec{D}$ is related to the electric field $\vec{E}$ by the relation

$$
\vec{D}=(\epsilon) \vec{E}
$$

where $(\boldsymbol{\epsilon})$ is the tensor dielectric constant. For an implied time factor exp $(i \omega t)$, it has the form

$$
(\epsilon)=\left(\begin{array}{ccc}
\epsilon^{\prime}-i q & 0 \\
i q & \epsilon^{\prime} & 0 \\
0 & 0 & \epsilon^{\prime \prime \prime}
\end{array}\right)
$$

${ }_{1}^{1}$ This work was carried out while the author was on a visit to the Laboratory for Electromagnetic Theory at the Technical University of Denmark.

${ }_{2}^{2}$ Figures in brackets indicate the literature references at the end of this paper. 
The quantities $\epsilon^{\prime}, \epsilon^{\prime \prime}$, and $q$ are functions of the density of the electrons and the ions and the frequency of collisions between them. They also depend, of course, on the strength of the applied magnetic field $H_{0}$ (see appendix).

The case usually considered is where the electromagnetic forces only influence the electrons. Furthermore, the motion of the ions is commonly neglected. For this situation, the properties of the plasma can be approximately described in a macroscopic sense in terms of the following quantities:

$$
\begin{aligned}
& \omega_{0} \text {, the (electron) plasma frequency } \\
& \omega_{T} \text {, the (electron) gyro frequency } \\
& \nu \text {, the effective collision frequency (for electrons). }
\end{aligned}
$$

The elements of the dielectric tensor are then given explicitly by

$$
\begin{aligned}
\frac{\epsilon^{\prime}}{\epsilon_{0}} & =1-\frac{i(\nu+i \omega) \omega_{0}^{2} / \omega}{\omega_{T}^{2}+(\nu+i \omega)^{2}} \\
\frac{q}{\epsilon_{0}} & =\frac{-\omega_{T} \omega_{0}^{2} / \omega}{\omega_{T}^{2}+(\nu+i \omega)^{2}} \\
\frac{\epsilon^{\prime \prime}}{\epsilon_{0}} & =1-\frac{i \omega_{0}^{2}}{(\nu+i \omega) \omega}
\end{aligned}
$$

The preceding can be generalized to include the influence of heavy ions by simply adding a summation prefix to the ratios on the right-hand side of equations (3), (4), and (5). Then, in each term, the appropriate value of $\omega_{0}, \nu$, and $\omega_{T}$ must be employed. This approach is valid for a weakly ionized medium and has been employed by Hines [8]. In the case of a plasma consisting of a neutral mixture of electrons, one type of heavy ions and a relatively large number of neutral molecules, the elements of the dielectric tensor have the form

$$
\begin{gathered}
\frac{\epsilon^{\prime}}{\epsilon_{0}}=1-\frac{i(\nu+i \omega) \omega_{0}^{2} / \omega}{\omega_{T}^{2}+\left(\nu_{e}+i \omega\right)^{2}}-\frac{i\left(\nu_{i}+i \omega\right) \hat{\mu} \omega_{0}^{2} / \omega}{\hat{\mu}^{2} \omega_{T}^{2}+\left(\nu_{i}+i \omega\right)^{2}}, \\
\frac{q}{\epsilon_{0}}=-\frac{\omega_{T} \omega_{0}^{2} / \omega}{\omega_{T}^{2}+\left(\nu_{\mathrm{e}}+i \omega\right)^{2}}+\frac{{ }^{2} \omega_{T} \omega_{0}^{2} / \omega}{\hat{\mu}^{2} \omega_{T}^{2}+\left(\nu_{i}+i \omega\right)^{2}}, \\
\frac{\epsilon^{\prime \prime}}{\epsilon_{0}}=1-\frac{i \omega_{0}^{2}}{\left(\nu_{e}+i \omega\right) \omega}-\frac{i \hat{\mu} \omega_{0}^{2}}{\left(\nu_{i}+i \omega\right) \omega} .
\end{gathered}
$$

where

$$
\hat{\mu}=\frac{m_{e}}{m_{i}}=\frac{\text { mass of electron }}{\text { mass of ion }}
$$

and $\nu_{e}$ and $\nu_{i}$ are the collision frequencies of the electrons and the ions, respectively, with the uncharged molecules. The effect of collisions between the electrons and the ions is neglected. $\omega_{0}$ and $\omega_{T}$ are the (electron) plasma and (electron) gyro frequencies. Since the charge of the ions is equal and opposite to the electrons, it follows that $\hat{\mu} \omega_{0}$ and $-\hat{\mu} \omega_{T}$ are the (ion) plasma and (ion) gyro frequencies, respectively.

It can be seen that if $\nu_{i}<<\omega$, the denominators in the second factors for $\epsilon^{\prime}$ and $q$ behave as $\hat{\mu}^{2} \omega_{T}^{2}-\omega^{2}$. Thus, at frequencies near the (ion) gyro frequency, the influence of the ions may be significant even though the mass ratio $\hat{\mu}$ is very small. However, when collisions are not negligible, the effect of the ions is usually quite small. In particular, if $\nu_{i}>>\omega$ and $\nu_{e}>>\omega$, this fact can be demonstrated by rewriting the elements of the dielectric tensor in the form

$$
\begin{gathered}
\frac{\epsilon^{\prime}}{\epsilon_{0}}=1-\frac{i \nu_{e} \omega_{0}^{2} / \omega}{\omega_{T}^{2}+\nu_{e}^{2}}\left[1+\frac{\nu_{i}}{\nu_{e}} \hat{\mu} \frac{\omega_{T}^{2}+\nu_{e}^{2}}{\hat{\mu}^{2} \omega_{T}^{2}+\nu_{i}^{2}}\right] \\
\frac{q}{\epsilon_{0}}=-\frac{\omega_{T} \omega_{0}^{2} / \omega}{\omega_{T}^{2}+\nu_{e}^{2}}\left[1-\hat{\mu}^{2} \frac{\omega_{T}^{2}+\nu_{e}^{2}}{\hat{\mu}^{2} \omega_{T}^{2}+\nu_{i}^{2}}\right]
\end{gathered}
$$




$$
\frac{\epsilon^{\prime \prime}}{\epsilon_{0}}=1-i \frac{\omega_{0}^{2}}{\nu_{e} \omega}\left[1+\frac{\nu_{e}}{\nu_{i}} \hat{\mu}\right]
$$

since the ratio $\left(\omega_{T}^{2}+\nu_{e}^{2}\right) /\left(\hat{\mu}^{2} \omega_{T}^{2}+\nu_{i}^{2}\right)$ is of the order of unity in most cases of practical interest. The square bracket terms may be replaced by unity since $\hat{\mu}$ is of the order of $10^{-4}$.

When the plasma becomes strongly ionized, the situation is more complicated. The formalism is given in the appendix where the relative number of ions and electrons is unrestricted However, in the case of a fully ionized plasma the elements of the dielectric tensor are given by $[9,10]$

$$
\begin{gathered}
\frac{\epsilon^{\prime}}{\epsilon_{0}}=1+\frac{\omega_{0}^{2}(1+\hat{\mu})\left[\omega^{2}-\hat{\mu} \omega_{T}-i \omega \nu(1+\hat{\mu})\right]}{\Delta} \\
\frac{q}{\epsilon_{0}}=\frac{\omega \omega_{T} \omega_{0}^{2}\left(\hat{\mu}^{2}-1\right)}{\Delta} \\
\frac{\epsilon^{\prime \prime}}{\epsilon_{0}}=1-\frac{\omega_{0}^{2}(1+\hat{\mu})}{\omega \mid \omega-i \nu(1+\hat{\mu})]}
\end{gathered}
$$

where

$$
\Delta=\omega^{2} \omega_{T}^{2}(1-\hat{\mu})^{2}-\left[\omega^{2}-\hat{\mu} \omega_{T}^{2}-i \omega \nu(1+\hat{\mu})\right]^{2} .
$$

As before, $\hat{\mu}$ is the ratio of the masses of the electron and the ion. Also, $\omega_{0}$ and $\omega_{T}$ are the (electron) plasma and (electron) gyro frequencies, respectively. In this case, however, $\nu$ is the collision frequency between the electrons and the ions which have equal and opposite charge.

It can be immediately seen that if $\hat{\mu}$ is set equal to zero, these expressions reduce to the form of equations (3), (4), and (5), which are derived under somewhat different conditions. It can also be seen that equations (12), (13), and (14) have the same form as (6), (7), and (8) if $\nu=\nu_{e}=\nu_{i}=0$. However, when collisions are non-negligible there is a fundamental difference between a weakly and a fully ionized plasma. This is a consequence of the coupling between the equations of motion for the electrons and the ions (see appendix).

Under the very reasonable approximation that $1 \pm \hat{\mu}$ can be replaced by 1 , it readily follows that eqs (12), (13), and (14) can be written in the form

$$
\begin{gathered}
\frac{\epsilon^{\prime}}{\epsilon_{0}}=1-\frac{i(\bar{\nu}+i \omega) \omega_{0}^{2} / \omega}{\omega_{T}^{2}+(\bar{\nu}+i \omega)^{2}} \\
\frac{q}{\epsilon_{0}}=-\frac{\omega_{T} \omega_{0}^{2} / \omega}{\omega_{T}^{2}+(\bar{\nu}+i \omega)^{2}} \\
\frac{\epsilon^{\prime \prime}}{\epsilon_{0}}=1-\frac{i \omega_{0}^{2}}{(\nu+i \omega) \omega}
\end{gathered}
$$

where $\bar{\nu}=\nu-i \hat{\mu} \omega_{T}^{2} / \omega=\nu\left(1-i \frac{\hat{\mu} \omega_{T}^{2}}{\nu \omega}\right)$

The quantity $\bar{\nu}$ could be described as an effective (complex) collision frequency. It should be noted that only the elements $\epsilon^{\prime}$ and $q$ of the dielectric tensor involve $\dot{\nu}$; the remaining element $\epsilon^{\prime \prime}$ is not changed.

It is now evident that the motion of the ions, for a fully ionized gas, can be neglected only if

$$
\hat{\mu}^{\prime} \omega_{T}^{2}<<\nu
$$

For low frequencies and/or low collision frequencies this condition may well be violated. 


\section{Field Equations}

Maxwell's equations in a source free region with a (tensor) dielectric constant $(\epsilon)$ and a (scalar) magnetic permeability $\mu$ are given by

$$
i(\epsilon) \omega \vec{E}=\operatorname{curl} \vec{H}
$$

and

$$
-i \mu \omega \vec{H}=\operatorname{curl} \vec{E} .
$$

It is desirable to write the first of these in the form

$$
i \omega \vec{E}=\left(\epsilon^{-1}\right) \operatorname{curl} \vec{H}
$$

where $\left(\boldsymbol{\epsilon}^{-1}\right)$ is the inverse of the dielectric tensor. It is not difficult to show that

$$
\epsilon_{0}\left(\epsilon^{-1}\right)=\left(\begin{array}{ccl}
M & -i K & 0 \\
i K & M & 0 \\
0 & 0 & \epsilon_{0} / \epsilon^{\prime \prime}
\end{array}\right)
$$

where

$$
M=\frac{\epsilon^{\prime} \epsilon_{0}}{\left(\epsilon^{\prime}\right)^{2}-q^{2}} \quad \text { and } \quad K=-\frac{y^{\epsilon_{0}}}{\left(\epsilon^{\prime}\right)^{2}-q^{2}} .
$$

This formula is quite general. The only restriction is that the z-axis is to be taken in the direction of the impressed magnetic field. In the following it is assumed that the fields do not vary in the z-direction. In terms of Cartesian coordinates, Maxwell's equations are then written

$$
\begin{gathered}
i \epsilon_{0} \omega E_{x}=M \frac{\partial H_{z}}{\partial y}+i K \frac{\partial H_{z}}{\partial x} \\
i \epsilon_{0} \omega E_{y}=i K \frac{\partial H_{z}}{\partial y}-M \frac{\partial H_{z}}{\partial x} \\
i \epsilon_{0} \omega E_{z}=\left(\epsilon_{0} / \epsilon^{\prime \prime}\right)\left[\frac{\partial H_{y}}{\partial x}-\frac{\partial H_{x}}{\partial y}\right] \\
-i \mu_{0} \omega H_{x}=N \frac{\partial E_{z}}{\partial y} \\
-i \mu_{0} \omega H_{y}=-N \frac{\partial E_{z}}{\partial x} \\
-i \mu_{0} \omega H_{z}=N\left[\frac{\partial E_{y}}{\partial x}-\frac{\partial E_{x}}{\partial y}\right]
\end{gathered}
$$

where $N=\mu_{0} / \mu$.

It is a relatively simple matter to eliminate the transverse component of the fields from the preceding six equations. Thus

$$
\left[\frac{\partial^{2}}{\partial x^{2}}+\frac{\partial^{2}}{\partial y^{2}}+\frac{k^{2}}{M N}\right] H_{z}=0
$$


and

$$
\left[\frac{\partial^{2}}{\partial x^{2}}+\frac{\partial^{2}}{\partial y^{2}}+\frac{k^{2} \epsilon^{\prime \prime}}{N \epsilon_{0}}\right] E_{z}=0
$$

where $k=\left(\epsilon_{0} \mu_{0}\right)^{1 / 2} \omega=2 \pi /$ wavelength. These latter two equations are valid only if the magnetic permeability and the elements of the dielectric tensor are constant for at least a given region.

The fact that $H_{z}$ and $E_{z}$ individually satisfy a wave equation means that any solution to our problem can be regarded as the linear combination of two partial solutions. In the first of these, $E_{z}=0$ and in the second, $H_{z}=0$. Thus without any subsequent loss of generality, attention can be restricted to these cases. It should be emphasized that this decomposition into independent partial fields is valid only when the derivatives with respect to $\approx$ are zero. As we shall see, the solution for $H_{z}=0$ is relatively trivial since the constant magnetic field $H_{0}$ then has no influence (at least within the limits of magneto-ionic theory).

\section{Reflection Coefficient for a Plane Boundary Between Free Space and Plasma}

With respect to the Cartesian coordinate system, a homogeneous plasma occupies the space $y>0$ and while $y<0$ corresponds to free space, the constant and uniform magnetic field is parallel to the z-axis. A plane wave with harmonic time dependence (i.e., exp $(i \omega t)$ ) is incident from below as indicated in figure 1.

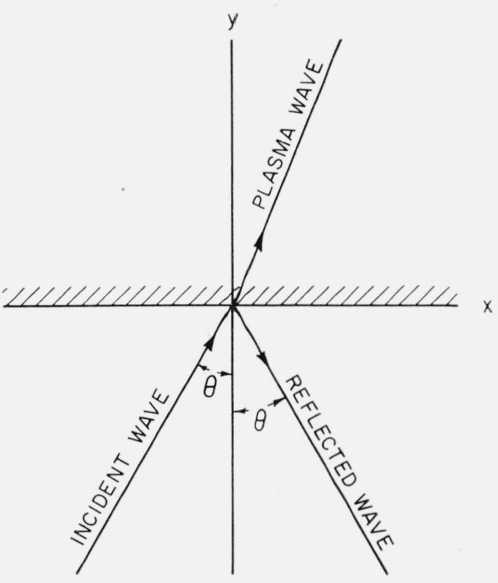

FIGURE 1. The coordinate system for reflection at a plane interface between a homogeneous plasma $(y>0)$ and free space $(y<0)$.

The constant magnetic field $\vec{H}_{0}$ is along the direction of the positive $z$-axis (out of the paper).

The angle of incidence is $\theta$ (measured to the negative $y$-axis) and the wave is polarized such that its magnetic field has only a component in the $z$-direction, denoted $H_{z}^{\text {inc }}$. Thus,

$$
H_{z}^{\mathrm{inc}}=h_{0} \exp (-i k C y) \exp (-i k S x)
$$

where $C=\cos \theta, S=\sin \theta$ and $h_{0}$ is a constant.

Since the reflected field $H_{z}^{\text {ref }}$ is a solution of the free-space wave equation and is to have the same dependence with $x$ as the incident wave, it must be of the form

$$
H_{z}^{\mathrm{ref}}=h_{0} R \exp (i k C y) \exp (-i k S x),
$$

where $R$ is by definition the reflection coefficient.

With similar reasoning, the solution for the plasma (i.e., $y>0$ ) must have the form

$$
H_{z}=f(y) \exp (-i k S x) \text {, }
$$


where $f(y)$ is some function of $y$. Furthermore, since $H_{z}$ is to satisfy eq $(27)$, it follows that $f(y)$ must satisfy

Solutions are of the form

$$
\left[\frac{\partial^{2}}{\partial y^{2}}+k^{2}\left(\frac{1}{M N}-S^{2}\right)\right] f(y)=0
$$

$$
\left.\exp \left[ \pm i k\left[(M N)^{-1}-S^{2}\right)\right]^{1 / 2} y\right]
$$

Since the plasma extends to $y=+\infty$, the solution corresponding to the negative sign in the exponent is pertinent. Therefore, the transmitted wave has the form

$$
H_{z}=h_{0} T \exp \left[-i k\left[(M N)^{-1}-S^{2}\right]^{1 / 2} y\right] \exp (-i k S x)
$$

where $T$ is by definition a transmission coefficient.

The boundary conditions are that the tangential components of the fields in the free space and in the plasma are to be continuous at $y=0$. Continuity of $H_{z}$ leads to

$$
1+R=T,
$$

and continuity of $E_{x}$, by virtue of eq (21), leads to

Thus,

$$
C(1-R)=T\left[\left[(M N)^{-1}-S^{2}\right]^{1 / 2} M+i K S\right] .
$$

$$
R=\frac{C-\Delta}{C+\Delta} \quad \text { and } T=\frac{2 C}{C+\Delta}
$$

where

$$
\Delta=M\left[(M N)^{-1}-S^{2}\right]^{1 / 2}+i K S .
$$

For an electron plasma where the motions of the ions are neglected, it is possible to write $\Delta$ in the following form:

$$
\Delta=\frac{\left[C^{2}+\frac{1+i L}{i L-L^{2}-\gamma^{2}}\right]^{1 / 2}\left(i L-L^{2}-\gamma^{2}\right)-i \gamma S}{(1+i L)^{2}-\gamma^{2}},
$$

where $L=(\nu+i \omega) \omega / \omega_{0}^{2}$, and $\nu=\omega_{T} \omega / \omega_{0}^{2}$. We have also set $N=1$ (i.e., $\mu=\mu_{0}$ ) although a plasma may be slightly diamagnetic.

The reflection coefficient, essentially in this form, was derived by Barber and Crombie [11] where the homogeneous electron plasma was to be an idealized representation for the ionosphere. Because of the assumption of a purely transverse magnetic field $H_{0}$, the horizontal direction of propagation is along the magnetic equator. For propagation from east-to-west, $S$ is positive, while for propagation from west-to-east $S$ is negative. $\gamma$ is then a positive real quantity.

For applications at low and very low radio frequencies, $\nu>>\omega$ so that to a good approximation

$$
L \cong \omega / \omega_{r} \quad \text { where } \quad \omega_{r}=\omega_{0}^{2} / \nu
$$

Some numerical results based on eq (37) are available [12].

\section{Reflection from a Stratified Plasma}

We shall now undertake to generalize the previous result to a plasma medium which is stratified in layers all parallel to the free-space interface at $y=0$. The situation is shown in figure 2 , where $P$ parallel layers are indicated. The $p$ th layer from the bottom is of thickness $l_{p}$ and its electrical properties are described by $M_{p}, N_{p}$, and $K_{p}$. The index $p$ ranges from 1 to $P$. It should also be noted that $l_{P}=\infty$. 


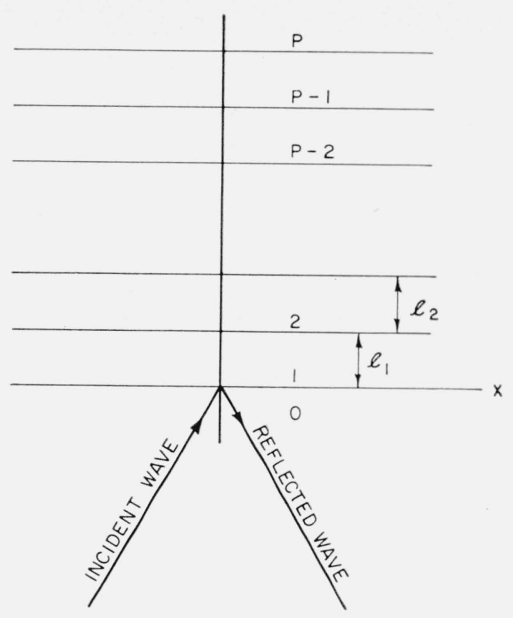

Figure 2. Reflection from a stratified plasma.

Again taking the incident wave to be polarized with its magnetic vector parallel to the $z$-axis, it is seen that the field for $y<0$ has the form

$$
H_{2}=h_{0}[\exp (-i k C y)+R \exp (i k C y)] \exp (-i k S x) .
$$

The problem is to find an expression for $R$ which involves the properties of the individual layers. It is possible, of course, to formally extend the results for the semi-infinite case by writing the solution in each layer as a linear combination of the two elementary forms given by (33). The two unknown coefficients for each layer are then found from the two boundary conditions at each plane interface. The resulting $2 N$ linear equations may then be solved in a straightforward but a very tedious manner for any specified but finite value of $N$. The resultant solution can be found in a more systematic way if the analogy with Schelkunoff's [13] theory of nonuniform transmission lines is exploited. We use this method here.

The wave impedances for the $p$ th layer are defined by

$$
K_{p}^{+}=-\frac{E_{x}^{+}}{H_{z}^{+}} \quad \text { and } \quad K_{p}^{-}=\frac{E_{x}^{-}}{H_{x}^{-}} .
$$
where

The superscript + signifies that the fields vary with $y$ according to the factor $\exp \left[-\imath \beta_{p} y\right]$

$$
\beta_{p}=k\left[\left(M_{p} N_{p}\right)^{-1}-S^{2}\right]^{1 / 2},
$$

whereas the superscript - signifies that the fields vary with $y$ according to the factor exp $\left[i \beta_{p} y\right]$. In the present case, the superscript + signifies a wave traveling in the positive $y$ direction (i.e., away from the interface) and the - signifies a wave traveling in the negative $y$-direction.

From eqs (41a) and (41b) it readily follows that the wave impedances are

$$
K_{p}^{+}=\eta_{0}\left(M_{p} \beta_{p}+i K_{p} S\right)
$$

and

$$
K_{p}^{-}=\eta_{0}\left(M_{p} \beta_{p}-i K_{p} S\right)
$$

where $\eta_{0}=\left(\mu_{0} / \epsilon_{0}\right)^{1 / 2} \cong 120 \pi$. The index $p$ ranges from 1 to $P$. Because of the quantity $K_{p}$ it is seen that $K_{p}^{+}$and $K_{p}^{-}$are not equal as they would be in an isotropic medium. ${ }^{3}$

The reflection coefficients at the interface between the $(P-1)$ th and the $P$ th layer are now defined by

$$
R_{P-1}=\frac{H_{z}^{-}}{H_{z}^{+}} \quad \text { and } \quad r_{P-1}=\frac{E_{x}^{-}}{E_{x}^{+}}
$$

${ }^{3}$ No confusion should arise between the symbols $K_{p}^{*}$ and $\overline{K_{p}^{-}}$and $K_{p}$, since the superscripted quantities are used only for the wave impedances. 
where the field components are evaluated within the $(P-1)$ th layer. Thus,

$$
R_{P-1}=\frac{K_{P-1}^{+}-K_{P}^{+}}{K_{P-1}^{-}+K_{P}^{+}} \quad \text { and } \quad r_{P-1}=\frac{1 / K_{P-1}^{+}-1 / K_{P}^{+}}{1 / K_{P-1}^{-}+1 / K_{P}^{+}}
$$

The reflection coefficients at the interface between the $(P-2)$ th and $(P-1)$ th layer are then

$$
R_{P-2}=\frac{K_{P-2}^{+}-Z_{P-1}}{K_{P-2}^{-}+Z_{P-1}} \quad \text { and } \quad r_{P-2}=\frac{1 / K_{P-2}^{+}-1 / Z_{P-1}}{1 / K_{P-2}^{-}+I / Z_{P-1}}
$$

where $Z_{P-1}$ is the impedance seen at the $(P-1)$ th interface. From analogy with transmission line theory

$$
Z_{P-1}=K_{P-1}^{+} \frac{1+r_{P-1} \exp \left(-i 2 \beta_{P-1} l_{P-1}\right)}{1+R_{P-1} \exp \left(-i 2 \beta_{P-1} l_{P-1}\right)}
$$

where $r_{P-1}$ and $R_{P-1}$ are given explicitly by eq (44). Now, in general,

$$
Z_{p}=K_{p}^{+} \frac{1+r_{p} \exp \left(-i 2 \beta_{p} l_{p}\right)}{1+R_{p} \exp \left(-i 2 \beta_{p} l_{p}\right)}
$$

so that the process may be continued until $p=1$, whence

Finally, for the bottom interface

$$
R_{1}=\frac{K_{1}^{+}-Z_{2}}{K_{1}^{-}+Z_{2}}
$$

which may be rewritten

$$
R_{0}=\frac{K_{0}^{+}-Z_{1}}{K_{0}^{-}+Z_{1}}
$$

$$
R=\frac{C-\Delta}{C+\Delta}
$$

where $\Delta=Z_{1} / \eta_{0}$ since $K_{0}^{+}=K_{0}^{-}=\eta_{0} C$.

For the special case of a two-layered plasma (i.e., $l_{2}=\infty$ ), the explicit expression for $\Delta$ becomes

with

$$
\Delta=\left(M_{1} \beta_{1}+i K_{1} S\right) \frac{1+r_{1} \exp \left(-i 2 \beta_{1} l_{1}\right)}{1+R_{1} \exp \left(-i 2 \beta_{1} l_{1}\right)}
$$

and

$$
R_{1}=\frac{\left(M_{1} \beta_{1}+i K_{1} S\right)-\left(M_{2} \beta_{2}+i K_{2} S\right)}{\left(M_{1} \beta_{1}-i K_{1} S\right)+\left(M_{2} \beta_{2}+i K_{2} S\right)}
$$

$$
r_{1}=\frac{1 /\left(M_{1} \beta_{1}+i K_{1} S\right)-1 /\left(M_{2} \beta_{2}+i K_{2} S\right)}{1 /\left(M_{1} \beta_{1}-i K_{1} S\right)+1 /\left(M_{2} \beta_{2}+i K_{2} S\right)} .
$$

The limiting case of a homogeneous plasma is recovered by letting $l_{1} \rightarrow \infty$, whence

$$
\Delta=M_{1} \beta_{1}+i K_{1} S,
$$

which is identical to eq (38) after dropping the subscript 1.

\section{Scattering from a Cylindrical Plasma Column}

In certain applications the plasma may be in the form of a cylindrical column. Examples are the ionization associated with meteor trails in the upper atmosphere and the ionization associated with the shock wave emanating from an exploding wire. In this section expressions for the reflected or scattered fields are derived under the assumption that the ionized column is infinite in length. First, the column is assumed to be homogeneous, but later the solution is generalized to allow for variation of the plasma properties in the radial direction.

Choosing a conventional cylindrical coordinate system $(\rho, \phi, z)$, a homogeneous plasma column occupies the space $\rho<a$. A constant magnetic field, $H_{0}$, exists through the plasma 
FIgURE 3. A magnetic line source at $\left(\rho_{0}, \phi_{0}\right)$ in the presence of a homogeneous cylindrical plasma column.

The constant magnetic field $H_{0}$ is directed along the positive $z$-axis (out of the paper).

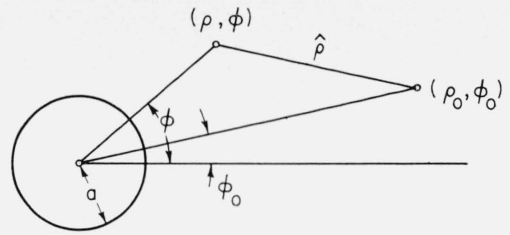

column and it is directed along the $z$-axis. The medium surrounding the cylinder, $\rho>a$, is taken to be free space. The source of the field is taken to be a line source located at $\rho=\rho_{0}$ and $\phi=\phi_{0}$ and running parallel to the $z$-axis and the plasma column. The line source radiates a cylindrical wave polarized so that its magnetic field has only a z-component.

The adoption of a line source rather than a plane wave source has the advantage of obtaining a more general solution. When $\rho_{0} \rightarrow \infty$, the incident wave has a plane front but in most practical applications the incident wave front is curved corresponding to a finite value of $\rho_{0}$.

The primary or incident field is given by [14]

$$
H_{x}^{\mathrm{inc}}=\frac{\epsilon_{0} \omega I}{4} H_{0}^{(2)}(k \hat{\rho})
$$

where $I$ is the strength of the line source (actually, it is the magnetic current), and where $H_{0}^{(2)}$ is the Hankel function of order zero of the second kind, and

$$
\hat{\rho}=\left[\rho_{0}^{2}+\rho^{2}-2 \rho \rho_{0} \cos \left(\phi-\phi_{0}\right)\right]^{1 / 2} .
$$

Employing an additional theorem for $H_{0}^{(2)}(k \hat{\rho})$, this can be written

$$
H_{z}^{\mathrm{ino}}=\frac{\epsilon_{0} \omega I}{4} \sum_{m=-\infty}^{+\infty} H_{m}^{(2)}\left(k \rho_{0}\right) J_{m}(k \rho) e^{-i m\left(\phi-\phi 0^{\prime}\right.}
$$

for $\rho<\rho_{0}$, where $H_{m}^{(2)}$ and $J_{m}$ are Hankel and Bessel functions of order $m$, respectively. When $\rho>\rho_{0}, \quad k \rho_{0}$ is to be interchanged with $k \rho$. The $\phi$-component of the primary magnetic field is then given by

$$
E_{\phi}^{\text {iue }}=\frac{i I}{4} \sum_{m=-\infty}^{+\infty} H_{m}^{(2)}\left(k \rho_{0}\right) k J_{m}^{\prime}(k \rho) e^{-i m\left(\phi-\phi_{0}\right)},
$$

where the prime indicates a differentiation with respect to the argument $k \rho$.

Since the scattered or reflected field $H_{z}^{s}$ outside the column is a solution of the wave equation, it can be written in the form [14]

$$
H_{z}^{s}=\frac{\epsilon_{3} \omega I}{4} \sum_{m=-\infty}^{+\infty} B_{m} H_{m}^{(2)}\left(k \rho_{0}\right) H_{m}^{(2)}(k \rho) e^{-i m\left(\phi-\phi_{0}\right)}
$$

where $B_{m}$ is an undetermined coefficient. The $\phi$ component of the scattered electric field is then

$$
E_{\phi}^{s}=\frac{i I}{4} \sum_{m=-\infty}^{+\infty} B_{m} H_{m}^{(2)}\left(k \rho_{0}\right) k H_{m}^{(2) \prime}(k \rho) e^{-i m\left(\phi-\phi_{0}\right)} .
$$

For the region $\rho<a$, the magnetic field $H_{z}$ satisfies

$$
\left[\frac{1}{\rho} \frac{\partial}{\partial \rho} \rho \frac{\partial}{\partial \rho}+\frac{1}{\rho^{2}} \frac{\partial^{2}}{\partial \phi^{2}}+\frac{k^{2}}{M N}\right] H_{Z}=0,
$$

which is just the wave equation. Therefore, we may write

$$
H_{Z}=\frac{\epsilon_{0} \omega I}{4} \sum_{m=-\infty}^{+\infty} A_{m} H_{m}^{(2)}\left(k \rho_{0}\right) J_{m}(\beta \rho) e^{-i m\left(\phi-\phi_{0}\right)}
$$


where $\beta=k(M N)^{-1}$, and $A_{m}$ is an undetermined coefficient. Since

it follows that

$$
i \epsilon_{0} \omega E_{\phi}=i K \frac{\partial H_{Z}}{\rho \partial \phi}-M \frac{\partial H_{Z}}{\partial \rho}
$$

$$
E_{\phi}=\frac{i I}{4} \sum_{m=-\infty}^{+\infty} A_{m} H_{m}^{(2)}\left(k \rho_{0}\right)\left[-\frac{m K}{\rho} J_{m}(\beta \rho)+M \beta J_{m}^{\prime}(\beta \rho)\right] e^{-i m\left(\phi-\phi_{0}\right)} .
$$

The boundary conditions at the surface of the plasma cylinder may be written

$$
\left.\begin{array}{l}
H_{z}=H_{z}^{\mathrm{inc}}+H_{z}^{s} \\
E_{\phi}=E_{\phi}^{\mathrm{inc}}+E_{\phi}^{s}
\end{array}\right\} \rho=a .
$$

Application of these leads readily to

and

$$
A_{m}=\frac{J_{m}(k a)+B_{m} H_{m}^{(2)}(k a)}{J_{m}(\beta a)}
$$

$$
B_{m}=-\left[\frac{\left(\frac{M}{N}\right)^{1 / 2} \frac{J_{m}^{\prime}(\beta a)}{J_{m}(\beta a)}-\frac{m K}{k a}-\frac{J_{m}^{\prime}(k a)}{J_{m}(k a)}}{\left(\frac{M}{N}\right)^{1 / 2} \frac{J_{m}^{\prime}(\beta a)}{J_{m}(\beta a)}-\frac{m K}{k a}-\frac{H_{m}^{(2)}(k a)}{H_{m}^{(2)}(k a)}}\right] \frac{J_{m}(k a)}{H_{m}^{(2)}(k a)} .
$$

This is the exact solution of the problem. If the constant magnetic field $H_{0}$ is removed the results are identical to that of a dielectric cylinder in the presence of a line source [15]. In this limiting case, $K=0, M=\epsilon_{0} / \epsilon$ and $N=\mu_{0} / \mu$.

\section{Scattering from a Cylindrically Stratified Plasma}

The preceding results are now generalized to a cylindrical column of ionization which consists of $P$ concentric layers. The situation is illustrated in figure 4 . The incident cylindrical wave again emanates from a line source at $\left(\rho_{0}, \phi_{0}\right)$ and the field is to be observed at $(\rho, \phi$.) The wave impedances may now be defined by

$$
K_{m, p}^{+}=-\frac{E_{\phi, m}^{+}}{H_{z, m}^{+}} \quad \text { and } \quad K_{m, p}^{-}=\frac{E_{\phi, m}^{-}}{H_{z, m}^{-}}
$$

where + and-refer to the two independent wave solutions, proportional to $J_{m}\left(\beta_{p} \rho\right)$ and $H_{m}^{(2)}\left(\beta_{p} \rho\right)$ in the $p$ th layer. In view of the equation

it follows that

$$
i \epsilon_{0} \omega E_{\phi}=i K_{p} \frac{\partial H_{z}}{\rho \partial \phi}-M_{p} \frac{\partial H_{z}}{\partial \rho}
$$

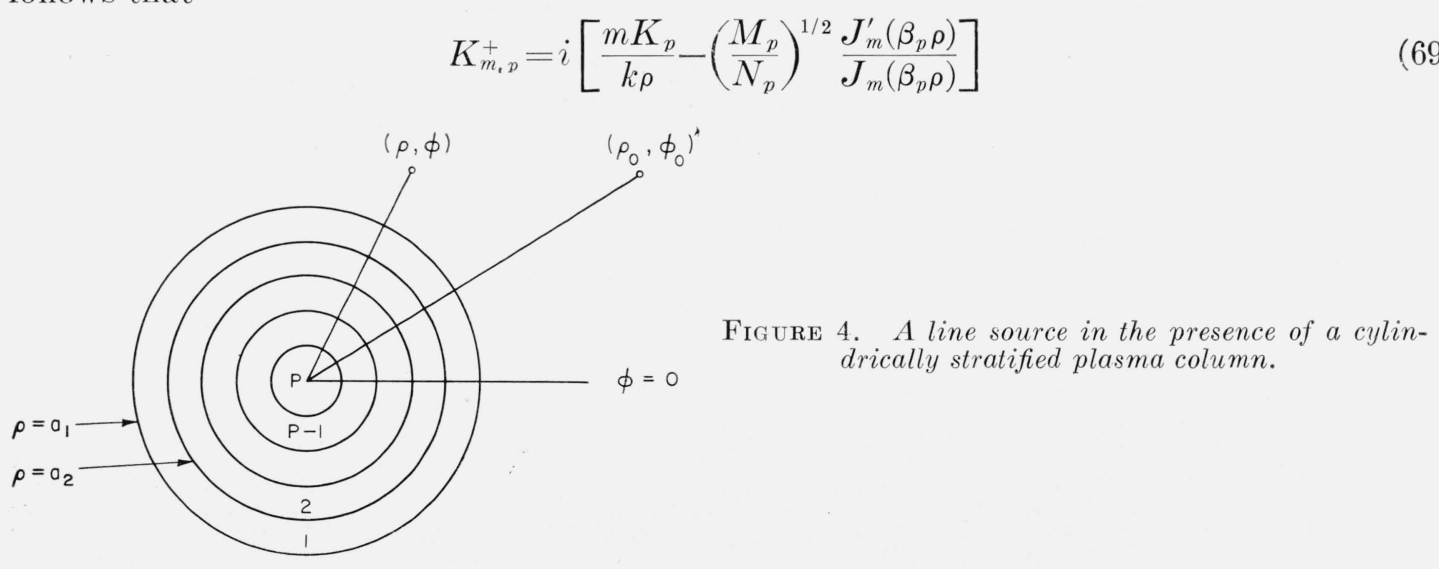


and

$$
K_{m, p}^{-}=-i\left[\frac{m K_{p}}{k \rho}-\left(\frac{M_{p}}{N_{p}}\right)^{1 / 2} \frac{H_{m}^{(2)}\left(\beta_{p} \rho\right)}{H_{m}^{(2)}\left(\beta_{p} \rho\right)}\right]
$$

The index $p$ ranges from 1 to $P$ to signify the appropriate concentric region in the plasma.

The reflection coefficients at the interface between the $p$ th and the $(p-1)$ th layer are then

$$
R_{m, p-1}=\frac{H_{z, m}^{-}}{H_{z, m}^{+}}=\frac{K_{m, p-1}^{+}-Z_{m, p}}{K_{m, p-1}^{-}+Z_{m, p}}
$$

and

$$
r_{m, p-1}=\frac{E_{\phi, m}^{-}}{E_{\phi, m}^{+}}=\frac{1 / K_{m, p-1}^{+}-1 / Z_{m, p}}{1 / K_{m, p-1}^{-}+1 / Z_{m, p}}
$$

where $Z_{m, p}$ is the impedance at the interface between the $p$ th and the $(p-1)$ th layer. $Z_{m, p}$ may be expressed in terms of the reflection coefficients $R_{m, p}$ and $r_{m, p}$ by again making use of Schelkunoff's nonuniform transmission line theory [13]. Thus,

$$
\begin{aligned}
Z_{m, p}=K_{m, p}^{+} \frac{1+r_{m, p} \chi_{m, e}^{+}\left(a_{p}, a_{p+1}\right) \chi_{m, e}^{-}\left(a_{p+1}, a_{p}\right)}{1+R_{m, p} \chi_{m, h}^{+}\left(a_{p}, a_{p+1}\right) \chi_{m, h}^{-}\left(a_{p+1}, a_{p}\right)}, \\
\chi_{m, e}^{+}\left(a_{p}, a_{p+1}\right)=\frac{E_{\phi, m}^{+}\left(a_{p+1}\right)}{E_{\phi, m}^{+}\left(a_{p}\right)}, \\
\chi_{m, e}^{-}\left(a_{p+1}, a_{p}\right)=\frac{E_{\phi, m}^{-}\left(a_{p}\right)}{E_{\phi, m}^{-}\left(a_{p+1}\right)}, \\
\chi_{m, h}^{+}\left(a_{p}, a_{p+1}\right)=\frac{H_{z, m}^{+}\left(a_{p+1}\right)}{H_{z, m}^{+}\left(a_{p}\right)}, \\
\chi_{m, h}^{-}\left(a_{p+1}, a_{p}\right)=\frac{H_{z, m}^{-}\left(a_{p}\right)}{H_{z, m}^{-}\left(a_{p+1}\right)} .
\end{aligned}
$$

The $\chi$ 's are clearly transmission factors which describe the fractional change of a wave as it propagates from one interface to the other within a layer. The numerator in eq (73) is thus a measure of the electric field at the $p$ th interface taking into account the transmission through the layer, reflection at the $(p+1)$ th layer and transmission back again to the $p$ th layer. The denominator of eq (73) corresponds, in a similar manner, to the magnetic field.

The specific form of the transmission factors is

$$
\begin{aligned}
& \chi_{m, e}^{+}\left(a_{p}, a_{p+1}\right)= \frac{M_{p} \beta_{p} J_{m}^{\prime}\left(\beta_{p} a_{p+1}\right)-m K_{p} J_{m}\left(\beta_{p} a_{p+1}\right) / a_{p+1}}{M_{p} \beta_{p} J_{m}^{\prime}\left(\beta_{p} a_{p}\right)-m K_{p} J_{m}\left(\beta_{p} a_{p}\right) / a_{p}}, \\
& \chi_{m, e}^{-}\left(a_{p+1}, a_{p}\right)= \frac{M_{p} \beta_{p} H_{m}^{(2) \prime}\left(\beta_{p} a_{p}\right)-m K_{p} H_{m}^{(2)}\left(\beta_{p} a_{p}\right) / a_{p}}{M_{p} \beta_{p} H_{m}^{(2) \prime}\left(\beta_{p} a_{p+1}\right)-m K_{p} H_{m}^{(2)}\left(\beta_{p} a_{p+1}\right) / a_{p+1}}, \\
& \chi_{m, h}^{+}\left(a_{p}, a_{p+1}\right)=\frac{J_{m}\left(\beta_{p} a_{p+1}\right)}{J_{m}\left(\beta_{p} a_{p}\right)},
\end{aligned}
$$

and

$$
\chi_{m, h}^{-}\left(a_{p+1}, a_{p}\right)=\frac{H_{m}^{(2)}\left(\beta_{p} a_{p}\right)}{H_{m}^{(2)}\left(\beta_{p} a_{p+1}\right)} .
$$

Then starting with the reflection coefficients $R_{m, P-1}$ and $r_{m, P-1}$, given by

$$
R_{m, P-1}=\frac{K_{m, P-1}^{+}-K_{m, P}^{+}}{K_{m, P-1}^{+}+K_{m, P}^{+}} \quad \text { and } \quad r_{m, P-1}=\frac{1 / K_{m, P-1}^{+}-1 / K_{m, P}^{+},}{1 / K_{m, P-1}^{+}+1 / K_{m, P}^{+}}
$$


we may obtain $R_{m, P-2}$ and $r_{m, P-2}$ which in turn enables us to obtain $R_{m, P-3}$ and $r_{m}, P-3$. The process is continued until the outermost interface is reached, whence

where

$$
R_{m, 0}=\frac{K_{m, 0}^{+}-Z_{m, 1}}{K_{m, 0}^{-}+Z_{m, 1}} \quad \text { and } \quad r_{m, 0}=\frac{1 / K_{m, 0}^{+}-1 / Z_{m, 1}}{1 / K_{m, 0}^{-}+1 / Z_{m, 1}}
$$

and

$$
K_{m, 0}^{+}=-i \frac{J_{m}^{\prime}(k \rho)}{J_{m}(k \rho)} \eta_{0}
$$

$$
K_{m, 0}^{-}=i \frac{H_{m}^{(2)}{ }^{\prime}(k \rho)}{H_{m}^{(2)}(k \rho)} \eta_{0}
$$

If the scattered field $H_{z}^{s}$ is written in the form

$$
H_{z}^{s}=\frac{\epsilon_{0} \omega I}{4} \sum_{m=-\infty}^{+\infty} B_{m} H_{m}^{(2)}\left(k \rho_{0}\right) H_{m}^{(2)}(k \rho) e^{-i m\left(\phi-\phi_{0}\right)},
$$

it follows that

$$
B_{m}=-R_{m, 0} \frac{J_{m}(k a)}{H_{m}^{(2)}(k a)} .
$$

It may be readily verified that $R_{m, 0}$ for the special case of a homogeneous plasma column (i. e., set $\left.a_{2}=0\right)$ is identical to the square bracket term on the right-hand side of eq $(66)$.

For purposes of computation it is convenient to locate the line source at a great distance from the cylindrical column in terms of wavelength. The Hankel functions of argument $k \hat{\rho}$ and $k \rho_{0}$ may then be replaced by the first term of their asymptotic expansions since $k \hat{\rho}$ and $k \rho_{0}>>1$. Thus,

$$
H_{z}^{\mathrm{inc}} \cong \frac{\epsilon_{0} \omega I}{4}\left(\frac{2 i}{\pi k \hat{\rho}}\right)^{1 / 2} e^{-i k \hat{\rho}}
$$

and

$$
H_{z}^{s} \cong \frac{\epsilon_{0} \omega I}{4}\left(\frac{2 i}{\pi k \rho_{0}}\right)^{1 / 2} e^{-i k \rho_{0}} \sum_{m=-\infty}^{+\infty} B_{m} e^{i m \pi / 2} H_{m}^{(2)}(k \rho) e^{-i m\left(\phi-\phi_{0}\right)} .
$$

This form of the solution would have been obtained directly if plane wave incidence was assumed at the outset. It should be noted that the factor preceding the summation in eq (89) is just the value of $H_{z}^{\text {inc }}$ evaluated at the center of the cylinder.

\section{A Note on the Other Polarization}

The results derived in the preceding sections are valid when the magnetic vector of the incident wave is parallel to the cylinder. The derivation for the other polarization, namely, that when the electric vector is parallel to the cylinder, is relatively trivial since the constant magnetic field $H_{0}$ has no influence. The dielectric constant is now simply a scalar and is equal to $\epsilon^{\prime \prime}$. The explicit results for $E$-parallel polarization may be obtained from those of $H$-parallel polarization by making the following transforms:

$$
\begin{aligned}
& H_{z} \rightarrow E_{\mathrm{z}} \\
& E_{x} \rightarrow-H_{x} \\
& E_{\phi} \rightarrow-H_{\phi} \\
& E_{y} \rightarrow-H_{y} \\
& E_{\rho} \rightarrow-H_{\rho} \\
& M \rightarrow N \\
& N \rightarrow M
\end{aligned}
$$

Also, of course, $K=0$ and now $M=\left(\epsilon_{0} / \epsilon^{\prime \prime}\right)$. 


\section{Appendix}

In this appendix a rather approximate theory of electric currents in a partially ionized gas is given. The gas is supposed to consist of electrons of mass $m_{e}$ and of charge $-e$; and positive ions of mass $m_{i}$ and charge $e$. Since the gas must be, to a close approximation, electrically neutral, the number of electrons and positive ions per unit volume is the same; each is taken equal to $N_{o}$. There are also $N_{a}$ neutral atoms or molecules per unit volume with mass $M_{i}$ indistinguishable from the positive ions. The electrons, positive ions and neutral atoms are regarded as three gases moving independently. The interaction between the electron and the ion gases is supposed to be smoothed into a continuous force. In fact only those forces are considered which result from macroscopic electromagnetic effects on charge and from frictionlike action and reaction between the free charges and the uncharged background. Only mean velocities and forces are employed, and non-linearities are avoided by a perturbation treatment.

Further assumptions, adopted here, are that the velocity of the gas as a whole is zero, and that the gradients of the electron and ion pressures are zero. The removal of these latter restrictions would require that the problem be treated on the basis of magneto hydrodynamics $[8,16]$. Such an approach has been given by Spitzer [7] for a wholly ionized gas and Cowling for a partially ionized gas [17].

The mean velocities of the electron gas, positive ion gas, and neutral gas are denoted by $\overrightarrow{v_{e}}, \overrightarrow{v_{i}}$, and $\overrightarrow{v_{a}}$, respectively. Since $m_{e} \ll m_{i}$, the electrons lose, on the average, a quantity of momentum equal to their mean momentum $m_{e}\left(\vec{v}_{e}-\vec{v}_{i}\right)$ at each collision with the charged ions. If the mean time between successive collisions is $\nu_{0}^{-1}$, then the momentum lost by electrons in collision with positive ions per unit volume is $N_{0} m_{e}\left(\overrightarrow{v_{e}}-\overrightarrow{v_{i}}\right) \nu_{0}$. Similarly, the momentum lost by electrons in collision with neutral atoms per unit volume is $N_{0} m_{e}\left(\overrightarrow{v_{e}}-\overrightarrow{v_{a}}\right) v_{e}$ where $\nu_{e}^{-1}$ is the mean time between successive collisions of electrons with neutral atoms. Remembering that the momentum of the mass as a whole is zero, and since the mass of the electrons is negligible, it follows that

and thus $\overrightarrow{v_{a}}=-\alpha \overrightarrow{v_{i}}$ where $\alpha=N_{0} / N_{a}$.

$$
N_{0} m_{i} \vec{v}_{i}+N_{\mathrm{a}} m_{i} \vec{v}_{\mathrm{a}}=0
$$

Now the electromagnetic forces acting on an electron are $-e\left(\vec{E}+\vec{\mu}_{0} \vec{v}_{e} \times \vec{H}_{0}\right)$ where $\vec{E}$ is the electric field of the wave and $\vec{H}_{0}$ is the constant magnetic field superimposed on the system. (It is assumed that $\vec{H}_{0}$ is much greater than the magnetic field $\vec{H}$ associated with the wave.) The equation of motion for the electron gas may then be written

$$
m_{e} \overrightarrow{d v_{e}} / d t=m_{e} i \omega \overrightarrow{v_{e}}=-e\left[\vec{E}+\mu_{0} \vec{v}_{e} \times \vec{H}_{0}\right]-m_{e} \nu_{0}\left(\overrightarrow{v_{e}}-\overrightarrow{v_{i}}\right)-m_{e} \nu_{e}\left(\vec{v}_{e}+\alpha \vec{v}_{i}\right)
$$

The equation of motion for the positive ions can be obtained in an equally simple fashion. In this case, the momentum lost by the positive ions in collision with the electrons is

$$
\left.-N_{0} m_{e} \overrightarrow{(v}_{e}-\vec{v}_{i}\right) \nu_{0}
$$

being equal and opposite to the quantity appearing above. The positive ions, in collision with the neutral atoms of the same mass, lose half their momentum relative to the neutral gas. Specifically, the momentum lost is $1 / 2 N_{0} m_{i} v_{i}(1+\alpha) \nu_{i}$ where $\nu_{i}^{-1}$ is the mean time between successive collisions of positive ions with neutral atoms. Therefore, the desired equation is

$$
m_{i} d \overrightarrow{v_{i}} / d t=m_{i} i \omega \overrightarrow{v_{i}}=e\left[\vec{E} \overrightarrow{+} \mu_{0} \vec{v}_{i} \overrightarrow{\times} H_{0}\right]+m_{e} \nu_{0}\left(v_{e}-\overrightarrow{-} v_{i}\right) \overrightarrow{-}-m_{i} \nu_{i} v_{i}(\overrightarrow{1}+\alpha) / 2 .
$$

Equations (91) and (92) may be solved for $\vec{v}_{e}$ and $\vec{v}_{i}$ in terms of $\vec{E}$ and other known quantities. The current density resulting from motion of the electrons and the ions is then equal to $N_{0} e\left(\overrightarrow{v_{i}}-\overrightarrow{v_{e}}\right)$. Noting that the displacement current is $i \epsilon_{0} \omega \vec{E}$ and the total current is $i(\epsilon) \omega \vec{E}$, it is seen that the dielectric tensor $(\epsilon)$ can be obtained from the relation 


$$
i(\epsilon) \omega \vec{E}=i \epsilon_{0} \omega \vec{E}+N_{0} e\left(\vec{v}_{i}-\vec{v}_{e}\right) .
$$

Choosing $\vec{H}_{0}$ to be directed along the z-axis, the dielectric tensor is found to have the form

$$
(\epsilon)=\left(\begin{array}{ccc}
\epsilon^{\prime} & -i q & 0 \\
i q & \epsilon^{\prime} & 0 \\
0 & 0 & \epsilon^{\prime \prime}
\end{array}\right) .
$$

The quantities $\epsilon^{\prime}, \epsilon^{\prime \prime}$, and $q$ are functions of $m_{e}, m_{i}, \nu_{e}, \nu_{i}, \nu, N_{0}, N_{a}$, and $H_{0}$. Explicit results for certain special cases are given in the body of the paper. To simplify the notation there, the results are expressed in terms of the positive real quantities $\omega_{0}$ and $\omega_{T}$ which are defined by

and

$$
\omega_{0}^{2}=N_{0} e^{2} / \epsilon_{0} m_{e}
$$

$$
\omega_{T}=-\mu_{0} H_{0} e / m_{e}
$$

Also, in discussing certain special cases in the text, the subscript on $\nu$ is dropped.

I would likə to thank Mrs. Eileen Brackett for preparation of the manuscript and Mr. Kenneth P. Spies for his careful reading of the paper. The present work was performed under contract CSO and A58-40 with the Electronics Research Directorate, U.S. Air Force Cambridge Research Laboratories.

\section{References}

[1] R. F. Whitmer, Principles of microwave interactions with ionized media, Microwave J. 17 (Feb. 1959); 47 (Mar. 1959).

[2] J. A. Ratcliffe, Magneto-ionic theory and its applications (Cambridge Univ. Press, England, 1959).

[3] S. Chapman and T. G. Cowling, The mathematical theory of non-uniform gases (Cambridge Univ. Press, 1939).

[4] T. G. Cowling, Electrical Conductivity of an ionized gas in a magnetic field, with applications to the solar atmosphere and the ionosphere, Proc. Roy. Soc A183, 453 (1945)

[5] K. T. Compton and I. Langmuir, Electrical discharges in gases, Rev. Mod. Phys. 2, 124 (1930).

[6] A. M. Cravath, The rate at which ions lose energy in elastic collisions, Phys. Rev. 36, 248 (1930).

[7] L. Spitzer, Jr., Physies of fully ionized gases, (Interscience Publ., New York, 1956).

[8] C. O. Hines, Generalized magneto-hydrodynamic formulae, Proc. Camb. Phil. Soc. 49, Pt. 2, 299-307 (1953).

[9] V. L. Ginzburg, The theory of propagation of radio waves in the ionosphere [in Russian] (Gostekhizdat, 1948).

[10] Ya. B. Fainberg and M F. Gorbatenko, Electromagnetic waves in plasma situated in a magnetic field, J. Tech. Phys. USSR 29, No. 5, 549 (May 1959).

[11] N. F Barber and D. D. Crombie, VLF reflections from the ionosphere in the presence of a transverse magnetic field, Jour. Atmospheric Terrest. Phys. 16, 37 (Oct. 1959).

[12] J. R. Wait and N. F. Carter, Field strength calculations for ELF radio waves, NBS Tech. Note No. 52 (PB-161553) (Mar. 1960).

[13] S. A. Schelkunoff, Electromagnetic waves (D. Van Nostrand Co., Inc., New York, N.Y., 1943).

[14] J. R. Wait, Electromagnetic radiation from cylindrical structures (Pergamon Press, London and New York, 1959). (In particular, see appendix.)

[15] C. Froese and J. R. Wait, Calculated diffraction patterns of dielectric cylinders at centimetric wavelengths, Canad. J. Phys. 32, 775-781 (1954).

[16] H. Alfvén, Cosmical electrodynamics, (Clarendon Press, Oxford, England, 1950).

[17] T. G. Cowling, Magnetohydrodynamics (Interscience Publishers, New York, N.Y., 1957).

\section{Additional References}

W. H. Eggiman, Scattering of a plane wave on a ferrite cylinder, IRE Trans. M-TT 8, 440-445 (July, 1960). V.V. Nikolskii, Radioengineering and Electronics (U.S.S.R.) 3, 756-759 (1958).

H. Wilhelmsson, Scattering of electromagnetic waves by an electron bean and a dielectric cylinder, Doktorsavhandlingar vid Chalmers Tekniska Högskola, Nr. 18 (Göteborg, 1958).

The solution given in these papers is a special case of eq (61) when the source is allowed to recede to infinity (i.e., $\left.\rho_{\mathrm{o}} \rightarrow \infty\right)$.

(Paper 65B2-53) 